\title{
Feocromocitoma relacionado a mielolipoma
}

\section{Myelolipoma-related pheochromocytoma}

\author{
Oscar G. Pamo-Reyna,' Edwin Hidalgo-Arroyo,' \\ Mauricio Guerra-Raygada, ${ }^{2}$ Zenaida Lozano-Miranda ${ }^{3}$ \\ y César Chian-García
}

\begin{abstract}
Pamo-Reyna OG, Hidalgo-Arroyo E, GuerraRaygada M, Lozano-Miranda Z, Chian-García C. Feocromocitoma relacionado a mielolipoma. Rev Soc Peru Med Interna. 2019;32(3): I17-121. https://doi.org//0.36393/spmi.v32i3.480
\end{abstract}

\begin{abstract}
RESUMEN
Mujer de 42 años que ingresó por hipertensión arterial maligna. Se le halló una tumoración suprarrenal izquierda de característica grasa, con elevación de las metanefrinas en orina de 24 horas. Con el diagnóstico de feocromocitoma, se le realizó una adrenalectomía. Se halló un mielolipioma. Después, la presión arterial se normalizó y la excreción de metanefrinas en orina de 24 horas estuvieron dentro de valores normales.
\end{abstract}

Palabras clave: Hipertensión arterial maligna, Suprarrenal, Mielolipoma, Metanefrinas, Feocromocitoma.

\section{ABSTRACT}

A 42-year-old woman was admitted with malignant arterial hypertension, and a left adrenal tumor of fat characteristics was found, with elevated excretion of 24 hour-urine metanephrines. With the diagnosis of pheochromocytoma, she underwent an adrenalectomy. It was found a myelolipoma. Afterwards, the blood pressure was normal and the excretion of 24-hour urine metanephrines was normal too.

Keywords: malignant high blood pressure, adrenal, myelipoma, metanephrines, pheochromocytoma.

\section{INTRODUCCIÓN}

El feocromocitoma puede presentarse como hipertensión arterial sostenida o crisis hipertensivas con cefalea, palpitaciones, sudoración profusa y ansiedad. Es un tumor raro de las células cromafines, productoras de catecolaminas, de la médula suprarrenal en el $90 \%$ de los casos. El $10 \%$ restante está conformado por

I Médico internista. Servicio de Medicina interna, Hospital Nacional Arzobispo Loayza (HNAL), Lima. Facultad de Medicina, Universidad Peruana Cayetano Heredia (FM UPCH).

2 Médico cardiólogo. Servicio de Cardiología. HNAL, Lima.

3 Médico patólogo. Servicio de Patología, HNAL, Lima.

4 Médico patólogo. Servicio de Patología, HNAL, Lima. FM UPCH. dichos tumores de localización extraadrenal (base del cerebro, corazón, vejiga, etc.) y son denominados paragangliomas.Alrededordel $30 \%$ de feocromocitomas es de carácter hereditario y relacionado con un rasgo autosómico dominante. Algunos de estos forman parte de la neoplasia endocrina múltiple tipo $2 \mathrm{a}$ y $2 \mathrm{~b}$, de la enfermedad de von Hippel Lindau, la neurofibromatosis y el paraganglioma familiar. ${ }^{1}$

Ocasionalmente se han descrito casos de la asociación de mielipoma con hipertensión arterial o feocromocitoma. El mielolipoma es una neoplasia benigna de la corteza adrenal, compuesto por grasa y tejido mieloide, y es el segundo incidentaloma suprarrenal primario más común (6\%-16\%), después del adenoma suprarrenal, y la mayoría de veces no es funcionante.

Se presenta el caso de una paciente con un síndrome de feocromocitoma asociado a un mielipoma.

\section{PRESENTACIÓN DEL CASO}

Paciente mujer de 42 años, natural y procedente de Lima, con antecedentes de asma bronquial desde los 6 años de edad, con tratamiento ocasional con salbutamol inhalatorio; hipotiroidismo a los 14 años por enfermedad 


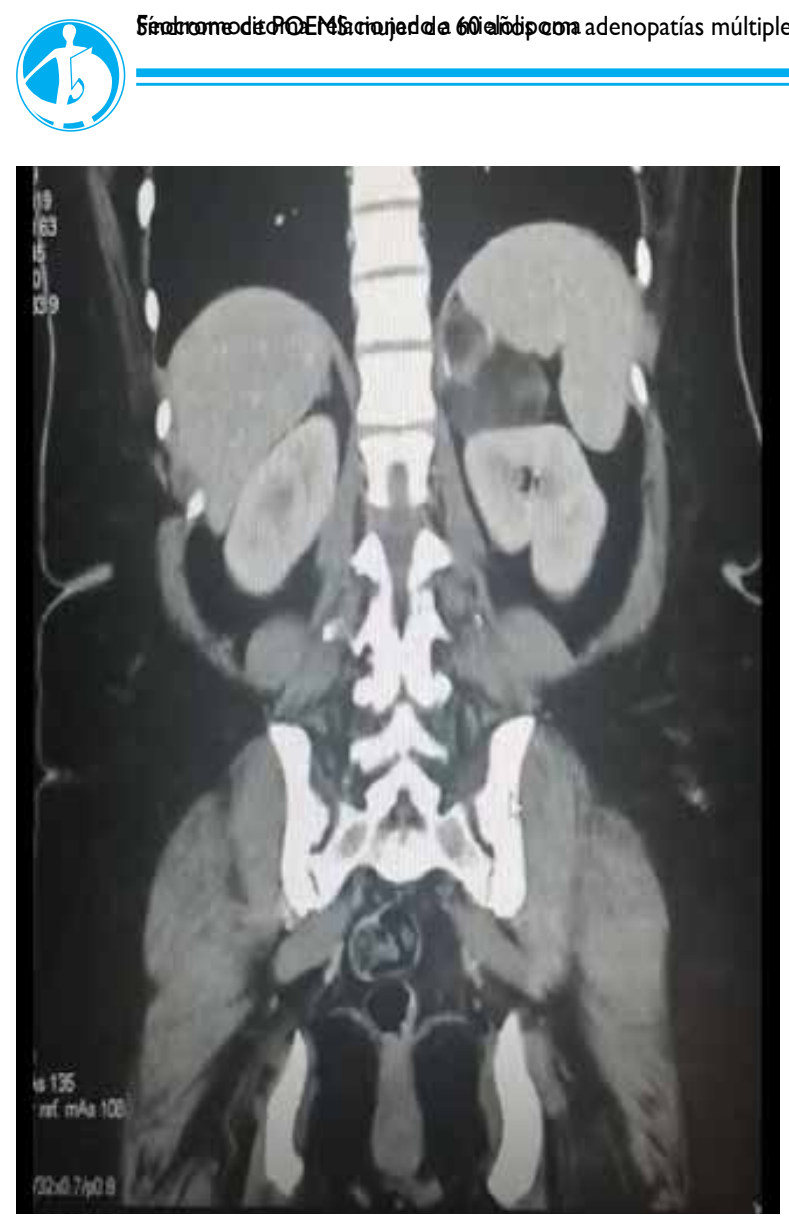

Figura I.Tumoración suprarrenal izquierda con densidad de tejido graso.

de Hashimoto, en tratamiento con levotiroxina, $100 \mu \mathrm{g} / \mathrm{d}$; "resistencia a la insulina" a los 20 años en tratamiento con metformina, $850 \mathrm{mg}$, dos veces/d. Se presentó con un mes de enfermedad, caracterizado por aparición de escotoma en el cuadrante temporal superior del ojo derecho, luego presentó escotoma central en campo visual izquierdo. Solo presentó cefalea frontal pulsátil de intensidad 7/10 el día previo al inicio de molestias visuales, que cedió con naproxeno, $550 \mathrm{mg}$, vía oral.

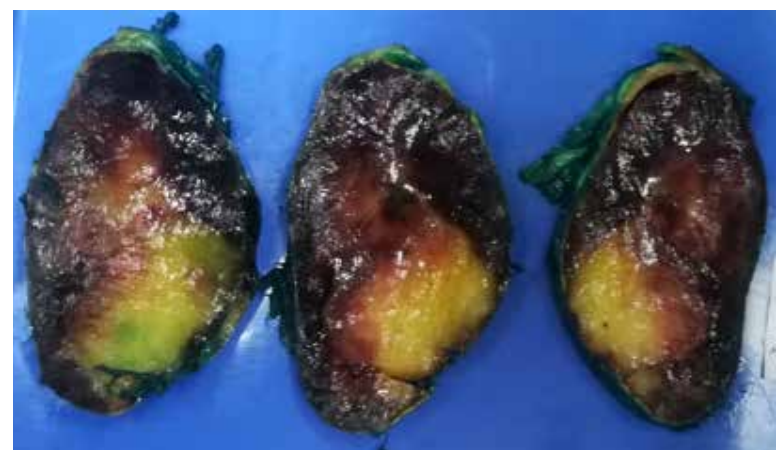

Figura 2. Cortes transversales de la tumoración suprarrenal.
Al ser evaluada se encontró PA 240/140 mmHg y frecuencia cardiaca de 85 latidos/min, y fue hospitalizada en UCI Coronarios, por emergencia hipertensiva. Durante su hospitalización, se controló la presión arterial tras la administración de nitroglicerina y labetalol; y, después recibió telmisartán, nifedipino y espironolactona.

\section{Examen físico}

IMC 32, tejido celular subcutáneo aumentado y de distribución centrípeta. Pulmones, cardiovascular y abdomen sin alteraciones. Fondo de ojo: hemorragias en astilla y manchas algodonosas; vasos retinianos de calibre disminuido a predominio arterial y edema de mácula.

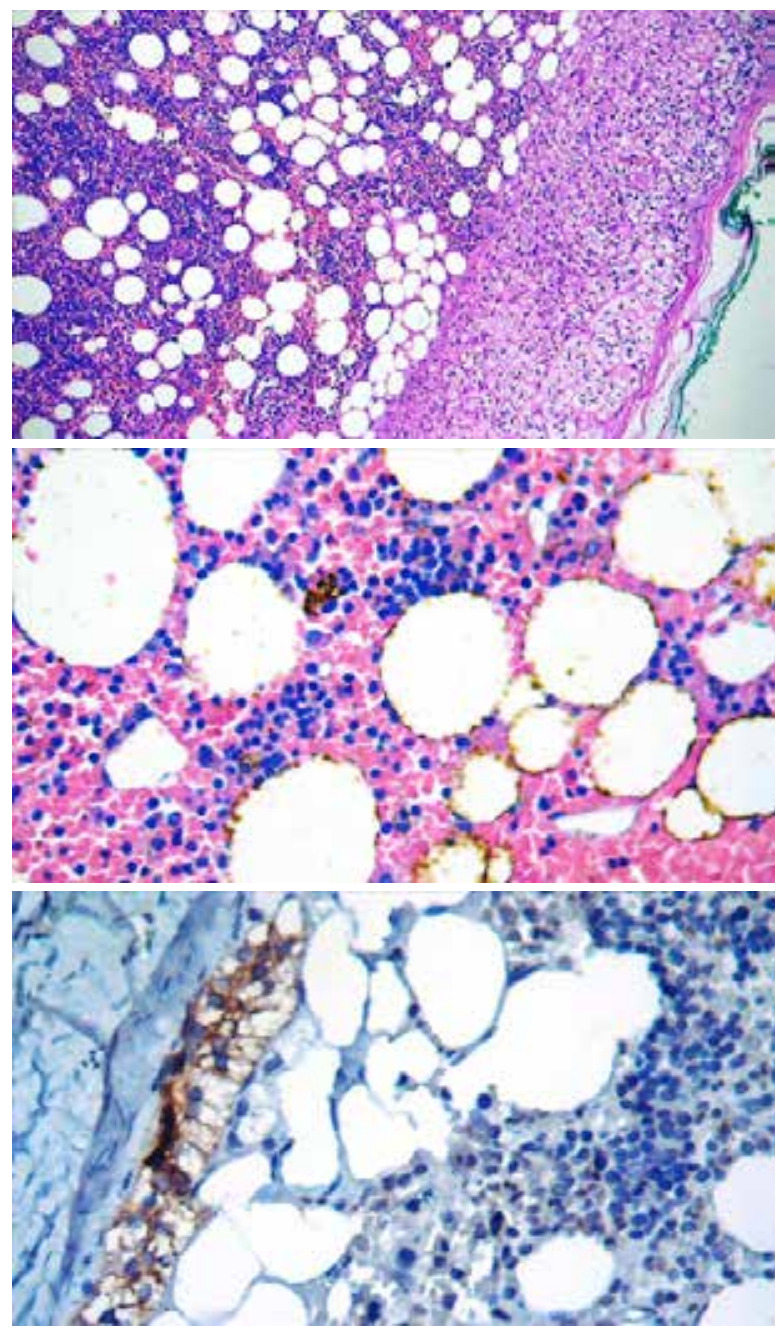

Figura 3.A) (HE, 4x) Hacia la derecha, remanente de corteza suprarrenal y, a la izquierda, tejido adiposo entremezclado con tejido hematopoyético. Las zonas más basofilicas corresponden a islas eritroides. $\mathrm{B}$ ) (HE, I0x). Nidos eritroides basofilicos, celdas adiposas, depósitos de hemosiderina (color marrón) y numerosos glóbulos rojos. C) Inmunohistoquímica (40x) con sinaptofisina, resaltando el componente neuroendocrino en medio del tejido adiposo. 


\section{Exámenes auxiliares}

Se encontró leve anemia microcítica e hipocrómica, y los valores de $\mathrm{HCO}_{3} 29 \mathrm{mEq} / \mathrm{L}, \mathrm{K} 2,6 \mathrm{mEq} / \mathrm{L}$, $\mathrm{Na} 133 \mathrm{mEq} / \mathrm{L}$ y Cl $95 \mathrm{mEq} / \mathrm{L}$.

ACTH 10,9 pg/mL (VN: 5,0-63,0 pg/mL) y cortisol a.m. 9,7 $\mu \mathrm{g} / \mathrm{dL}$ (VN: 6,2-26,0 $\mu \mathrm{g} / \mathrm{dL}$ ); aldosterona plasmática $15,4 \mathrm{ng} / \mathrm{dL}$ (primera hora de la mañana, en posición decúbito supino, VN: 2,94-16,1 ng/dL), actividad de renina plasmática $3,4 \mathrm{ng} / \mathrm{mL} / \mathrm{h}(\mathrm{VN}: 1,5-5,7 \mathrm{ng} / \mathrm{mL} / \mathrm{h}$ ) y aldosterona plasmática/actividad de renina plasmática (PAC/PRA) 4,52 (VN: 0,9-28,9),

Metanefrinas en orina de $24 \mathrm{~h}: 2,21 \mathrm{mg} / 24 \mathrm{~h}(\mathrm{VN}$ : $0,05-1,00 \mathrm{mg} / 24 \mathrm{~h})$ y metanefrinas/creatinina $1,58(\mathrm{VN}$ : $0-0,60)$.

Electrocardiograma: ritmo sinusal, crecimiento biauricular, hipertrofia ventricular izquierda y sobrecarga sistólica del ventrículo izquierdo.

Ecocardiografia: función sistólica de ambos ventrículos conservada, disfunción diastólica tipo 1, motilidad global y segmentaria conservada e hipertrofia concéntrica grave del ventrículo izquierdo.

Tomografía de abdomen: tumoración suprarrenal izquierda de $8 \mathrm{~cm}$ de diámetro, con densidad característica de tejido graso (Figura 1).

\section{Evolución}

Los niveles de $\mathrm{K}$, $\mathrm{Na}$ y $\mathrm{Cl}$ se corrigieron en los dos días posteriores solo con el control de la presión arterial, sin necesidad de reposición de estos electrólitos.

Se realizó la adrenalectomía laparoscópica, previo bloqueo alfa y beta, con terazocina y propranolol. La pieza macroscópica tuvo un peso de $150 \mathrm{~g}$, medía $8,7 \times 7,0 \mathrm{~cm} \times 4,0 \mathrm{~cm}$, con la superficie rodeada de grasa. Al corte, se evidenció un parénquima amarillento con extensas áreas hemorrágicas y calcificaciones adyacentes al tejido adiposo (Figura 2).

En el estudio anatomopatológico de la tumoración suprarrenal extraída se confirmó el diagnóstico de mielolipoma y no se distinguió otro tejido característico (Figuras 3).

La presión arterial se mantuvo normal en el posoperatorio inmediato, sin la administración de antihipertensivos, y el dosaje control de metanefrinas en orina fue de $0,098 \mathrm{mg} / 24 \mathrm{~h}$ (VN: 0,05-1,00 mg/24h).

\section{DISCUSIÓN}

La hipertensión arterial grave de la paciente más la retinopatía hipertensiva grado 4 llevaron al diagnóstico dehipertensión arterial maligna.Los estudios descartaron patología vascular renal, glomerulopatías, enfermedades autoinmunes, consumo de simpaticomiméticos y fármacos. Con foco en la hipertensión maligna de causa endocrinológica, los resultados de ACTH y cortisol descartaron hipercorticismo. Por la presencia inicial de hipopotasemia se planteó un hiperaldosteronismo primario pero los valores normales de aldosterona plasmática, de la actividad de renina plasmática y de la relación PAC/PARA menor de 300 ng/L fueron normales. ${ }^{4,5}$

Las metanefrinas en orina de $24 \mathrm{~h}$ y la relación metanefrinas/creatinina estuvieron elevadas; y, con metanefrinas en orina de $24 \mathrm{~h}>110,7 \mu \mathrm{g} / \mathrm{d}$, por lo que se alcanzaba una sensibilidad de $80 \%$ y especificidad de $82 \%$ para el diagnóstico de feocromocitoma. ${ }^{4,6,7}$

Por todo lo mencionado, el diagnóstico de feocromocitoma era la primera posibilidad; pero, lo que hallamos, desde el punto de vista de imágenes y de histopatología, fue una tumoración suprarrenal que correspondía a un mielolipoma.

El mielolipoma es una tumoración benigna, usualmente no funcionante, compuesto por tejido adiposo maduro y células hematopoyéticas, y que se encuentra en la lista de los tumores estromales y mesenquimatosos de la corteza adrenal según la última actualización de tumores endocrinos de la WHO. ${ }^{8}$ El mielolipoma rara vez pueden ser extraadrenal (mediastino, bazo, riñón, huesos, tórax, cavidad nasal, corteza suprarrenal ectópica, extradural o en los ojos).

El número de reportes de casos sobre mielolipomas suprarrenales ha aumentado considerablemente en las últimas décadas debido a los métodos de diagnóstico por imagen como TEM y RMN. En la tomografía, los valores de atenuación de -90 a -120 HU son característicos del tejido adiposo, y la presencia de densidad de grasa es útil y fundamental en el diagnóstico de una masa como un mielolipoma. ${ }^{8}$ En el diagnóstico diferencial radiológico hay que considerar el liposarcoma retroperitoneal y el angiomiolipoma extrarrenal.

Los mielolipomas pueden producir síntomas por el tamaño que pueden alcanzar, provocando molestias vagas, dolor o compresión de estructuras vecinas. 
Se pueden asociar a hipertensión arterial, obesidad, diabetes, aterosclerosis y neoplasia maligna; y, el $10 \%$ puede coexistir con desórdenes endocrinológicos como hiperplasia adrenal congénita, virilización simple, deficiencia de 21-hidroxilasa, de 17-alfahidroxilasa y, más raro, deficiencia de 11-betahidroxilasa, que se manifiestan como hipercortisolismo, hiperaldosteronismo e hiperandrogenismo, que desaparecen con la extirpación del tumor, por lo que se tratarían de mielolipomas funcionantes. ${ }^{9,10}$

Son escasos los reportes de mielolipomas asociados a feocromocitomas o hipersecreción de catecolaminas, así se tiene los siguientes:

- Una mujer de 39 años, con historia de cefalea e hiperhidrosis, con niveles elevados de norepinefrina en suero, y de dopamina, norepinefrina, normetanefrina y ácido vanilmandélico en orina de 24 horas; y, se halló dos tumores adrenales derechos: un mielolipoma de $23 \mathrm{~g}$ y un feocromocitoma de $48 \mathrm{~g}$, este último con elevadas concentraciones de aminas adrenérgicas. ${ }^{11}$

- Una mujer de 52 años con hipertensión de larga evolución con un incidentaloma suprarrenal izquierdo. Con el diagnóstico clínico presuntivo de feocromocitoma se realizó la adrenalectomía. El examen histopatológico reveló un tumor mixto de un mielolipoma grande con focos infiltrantes de médula suprarrenal. Después de la adrenalectomía, la presión arterial y las anomalías bioquímicas se normalizaron. $^{12}$

- Una paciente de 48 años con hipertensión arterial, tumoración adrenal derecha de 11,0 x 10,5 x 7,0 cm, y con metanefrinas elevadas en orina de $24 \mathrm{~h} 3,5$ veces por encima del límite superior normal; y, que luego de adrenalectomía, se confirmó mielolipoma, la presión arterial se controló y las metanefrinas se normalizaron. $^{13}$

- Una paciente de 53 años con hipertensión de larga data fue evaluada por dolor en el flanco izquierdo. La TAC abdominal reveló una masa suprarrenal izquierda de $2,8 \mathrm{~cm}$. Los niveles de metanefrina, normetanefrina y ácido vanilmandélico en una muestra de orina de $24 \mathrm{~h}$ estuvieron incrementados. La adrenalectomía reveló una masa nodular con una superficie roja al corte en la región medular, y médula agrandada. En el examen microscópico, la masa se componía de tejido adiposo maduro mezclado con tejido hematopoyético, con una médula hiperplásica con una relación cortico/medular de 1:5. Después de la cirugía, los niveles de presión arterial y catecolaminas se normalizaron. ${ }^{14}$

- Un varón de 68 años con hipertensión no controlada y masa suprarrenal. La RMN reveló una masa suprarrenal izquierda hemorrágica de $9 \mathrm{~cm}$ que contenía grasa. Se halló un aumento de las catecolaminas en suero y orina y VMA en orina de $24 \mathrm{~h}$ (normetanefrina en suero, $295 \mathrm{pg} / \mathrm{mL}$; normetanefrina en orina, $1586 \mu \mathrm{g} / 24 \mathrm{~h}$; VMA en orina de 24 h, 11,1 mg/24 h), que llevó al diagnóstico preoperatorio de feocromocitoma. A la macroscopia, la glándula suprarrenal estaba reemplazada (>95\%) por tumor hemorrágico y friable, de $11 \times 9,5 \times 7 \mathrm{~cm}$, con un borde no continuo de corteza suprarrenal en la periferia del tumor. A la microscopía, reveló un tumor mayormente hemorrágico compuesto de grasa madura con islas de células hematopoyéticas, compatible con mielolipoma suprarrenal. ${ }^{15}$

- Un varón de 58 años, con diabetes mellitus, hipertensión arterial, con dolor en hipocondrio y región lumbar izquierda, causado por una enorme tumoración suprarrenal izquierda de 18,4 x 10,1 cm, con características de mielolipoma en la TAC, con dosaje de ácido vanilmandélico en orina de $24 \mathrm{~h}$ de $80,89 \mathrm{mg} / \mathrm{d}$ (VN: 1,6-4,2 mg/d), quien luego de adrenalectomía se volvió normotenso, y el nuevo dosaje de ácido vanilmandélico fue de 4,13 mg/d, y en la patología se encontró solo mielolipoma. ${ }^{16}$

- Un paciente de 40 años con hipertensión arterial y tumoración adrenal derecha de $9,8 \times 8,5 \mathrm{~cm}$, con metanefrinas en orina de $24 \mathrm{~h}$ tres veces por encima del límite superior normal y que luego de adrenalectomía se confirmó mielolipoma en la histopatología y la inmunohistoquímica del espécimen reveló ser positivo para la cromogranina A, sugerente de gránulos secretores de catecolamina. ${ }^{17}$

El presente caso se trató de una paciente que se presentó como hipertensión arterial maligna y se le halló un mielolipoma suprarrenal izquierdo secretor de catecolaminas, demostrado por la elevada excreción de metanefrinas en orina de $24 \mathrm{~h}$.

En la histopatología, no se encontró un tumor ni hiperplasia de células cromafines, excepto algunas células dispersas que dieron tinción positiva para sinaptofisina. Luego de la adrenalectomía, la excreción urinaria de metanefrinas disminuyó a valores normales. 
Los mielolipomas son tumores en su mayoría benignos, y algunos pueden estar asociados a endocrinopatías o ser secretores de hormonas; y, de estos, pueden ser secretores de catecolaminas, lo que nos llevaría al diagnóstico de feocromocitoma producido por el mielolipoma, como sería nuestro caso.

Por lo expuesto anteriormente, el feocromocitoma es un síndrome antes que una entidad única, dado que tiene múltiples causas. Con respecto al mielolipoma, este puede asociarse con un tumor o con una hiperplasia de células cromafines o per se producir catecolaminas. Lo que falta explicar es la producción de catecolaminas por el mielolipoma.

La producción de catecolaminas ocurre en las células cromafines del tejido adrenal y en las neuronas pero también por las células inmunitarias activadas (linfocitos B y T y los macrófagos). Algunos estudios demuestran que el tejido adiposo blanco y pardo de las ratas poseen las enzimas para la producción de catecolaminas, independientemente de la inervación simpática, especialmente en situaciones de estrés. ${ }^{18-19}$ Esto llevaría a postular que una incrementada expresión de determinada alteración genética se traduce en una excesiva producción de catecolaminas por el tejido adiposo, como sería el caso del mielolipoma, causando el síndrome de feocromocitoma. Esto podría confirmarse midiendo la cantidad de catecolaminas en el tejido del mielolipoma, en ausencia de tumor o hiperplasia de células cromafines,

En conclusión, ante cualquier tumor suprarrenal con características grasas que sugiera un mielolipoma, debe hacerse el estudio correspondiente en búsqueda de que se trate de un tumor secretor de hormonas, específicamente secretor de catecolaminas, el que originaría un síndrome de feocromocitoma, como en el presente caso.

\section{REFERENCIAS BIBILIOGRÁFICAS}

I. Dahia P. Pheochromocytoma. Rar Disease database. URL disponible en: https://rarediseases.org/rare-diseases/pheochromocytoma/

2. Morales $E$, González R, Gutiérrez E, Gutiérrez-Solís E, et al. Hipertensión arterial maligna. Una visión actualizada. NefroPlus. 20I I;4(I):34-43.

3. Mahana D. Hipertensión arterial de origen endocrinológico. Rev Med Clin Condes. 2005; 16(2):93-99.

4. Petersenn S, Unger N, Walz MK, Mann K. Diagnostic value of biochemical parameters in the differential diagnosis of an adrenal mass.Ann NY Acad Sci. 2006;1073:348-357. doi: 10.1 196/annals. 1353.039

5. Thomas RM, Ruel E, Shantavasinkul PC, Corsino L. Endocrine hypertension: An overview on the current etiopathogenesis and management options. World J Hypertens. 2015;5(2):14-27.

6. Unger N, Pitt C, Schmidt IL, Walz MK, Schmid KW, Philipp T, et al. Diagnostic value of various biochemical parameters for the diagnosis of pheochromocytoma in patients with adrenal mass. European Journal of Endocrinology. 2006;154:409-4I7.

7. Carr JC, Spanheimer PM, Rajput M, Dahdaleh FS, Lal G, Weigel RJ et al. Discriminating pheochromocytomas from other adrenal lesions: the dilemma of elevated catecholamines.Ann Surg Oncol. 2013;20:3855-386I.

8. Decmann Á, Perge P, Tóth M, Igaz P. Decmann Á, Perge P et al. Adrenal myelolipoma: a comprehensive review. Endocrine. 2018;59:7-I5.

9. Adapa S, Naramala S, Gayam V, et al. Adrenal incidentaloma: Challenges in diagnosing adrenal myelolipoma. J Investig Med High Impact Case Rep. 2019;7:23247096198703। I. doi: I0.1 I77/23247096198703। I

10. Kodzo-Grey Venyo,A. Myelolipoma of the adrenal gland:A review and update of the literature. Pulsus J Surg Res. 2018;2(2):50-63.

II. Ukimura O, Inui E, Ochiai A, Kojima M, Watanabe H. Combined adrenal myelolipoma and pheochromocytoma.J Urol. 1995; I54(4): I470.

12. Ishay A, Dharan M, Luboshitzky R. Combined adrenal myelolipoma and medullary hyperplasia. Horm Res. 2004;62:23-26.

13. Tamidari H, Kumar Mishra A, et al. Catecholamine secreting adrenal myelolipoma. Letter to the editor. Indian J Med Sci. 2006;60(8):331-333.

14. Güresci S, Kara C, Ertugrul DT, Ünsal A. Combined adrenal medullary hyperplasia and myelolipoma: A mimicker of pheochromocytoma - Case Report. Turk Jem. 2009; 13:84-6.

15. Ferguson NL Gray K, Orucevic S. Adrenal myelolipoma masquerading as pheochromocytoma.Am J Clin Pathol. 2012; I38(Suppl I):A325, https://doi. org//0.1093/ajcp/I38.suppll.302

16. Udupa Sujatha, Usha M,Visweswara RN, et al. Left-sided giant adrenal myelolipoma secreting catecholamine. Indian J Pathol Microbiol. 2012;55:389-39I.

17. Jakka N,Venkateshwarlu J, Satyavani N, Neelaveni K, Ramesh J. Functioning adrenal myelolipoma: A rare cause of hypertension. Indian J Endocrinol Metab. 20 I3; I7(Suppl I):S249-S25I. doi:10.4103/2230-8210.II9588

18. Vargovic P,Ukropec J, Laukova M, Cleary S, et al Adipocytes as a new source of catecholamine production. FEBS Lett. 201 I ;585:2279-84.

19. Kvetnansky R, Ukropec J, Laukova M, Manz B, Pacak K, Vargovic P. Stress stimulates production of catecholamines in rat adipocytes. Cell Mol Neurobiol. 20 I2;32(5):80 I-8I3. doi: I0.1007/s I057I-0I2-9822-6

CORRESPONDENCIA: Edwin Hidalgo-Arroyo

edwin.hidalgo.a@gmail.com

FECHA DE RECEPCIÓN: 2 de setiembre de 2019.

FECHA DE ACEPTACIÓN: 20 de setiembre de 2019. 